\title{
Construção de tecnologia educativa para o autocuidado de pessoas com doença renal
}

\section{crônica em hemodiálise}

\author{
Construction of education technology for the self-care of people with chronic kidney disease in \\ hemodialysis \\ Construcción de tecnología educativa para el auto cuidado de personas con enfermedad renal \\ crónica en hemodiálisis
}

Recebido: 25/10/2022 | Revisado: 02/11/2022 | Aceito: 19/01/2022 | Publicado: 21/01/2022

\author{
Gabriela de Oliveira Benites \\ ORCID: https://orcid.org/0000-0002-4053-5347 \\ Universidade Federal do Rio Grande, Brasil \\ E-mail: gabiobenites@gmail.com \\ Paula Pereira de Figueiredo \\ ORCID: https://orcid.org/0000-0002-1875-7357 \\ Universidade Federal do Rio Grande, Brasil \\ E-mail: paulapfigueiredo@yahoo.com.br \\ Lenice Dutra de Sousa Canuso \\ ORCID: https://orcid.org/0000-0001-6436-0310 \\ Universidade Federal do Rio Grande, Brasil \\ E-mail: leniceds@hotmail.com \\ Fabiane Ferreira Francioni \\ ORCID: https://orcid.org/0000-0002-3384-0802 \\ Universidade Federal do Rio Grande, Brasil \\ E-mail: francionifabiane@gmail.com
}

\begin{abstract}
Resumo
O estudo teve como objetivo construir uma tecnologia educativa destinada ao autocuidado de pessoas com Doença Renal Crônica em tratamento hemodialítico sob a perspectiva da produção científica na área. Para tanto, foi realizada uma pesquisa de natureza qualitativa, do tipo descritiva e exploratória por meio de uma Revisão Integrativa. Subsequentemente construiu-se a tecnologia educativa a partir de um instrumento norteador. A coleta de dados ocorreu em janeiro de 2021 e, após a aplicação dos critérios de inclusão e exclusão, obteve-se um total de vinte e um artigos. Os resultados apontaram seis categorias temáticas mais prevalentes no que diz respeito às principais dificuldades enfrentadas por essa população a respeito de seu autocuidado, contudo, o material didático produzido integrou todas as temáticas identificadas, ainda que não tenham apresentado expressividade do ponto de vista quantitativo. A tecnologia educativa, produto deste estudo, constitui-se em uma série de cinco cartilhas que se configuram em subprodutos seriados, distintos e interdependentes. Percebe-se esse trabalho como relevante devido à temática estudada apresentar-se como um problema de saúde pública no Brasil e no mundo, que impacta na qualidade de vida das populações e que requer intervenções em saúde com vistas à promoção do autocuidado. Ademais, acredita-se que a produção do material educativo contribui para a divulgação de ações em saúde pautadas em evidências científicas e, diferente de outros formatos de produções acadêmicas, conversa diretamente com o público-alvo, oportunizando à população em geral o acesso ao conhecimento advindo de pesquisas na área da saúde.
\end{abstract}

Palavras-chave: Insuficiência renal crônica; Autocuidado; Diálise renal.

\begin{abstract}
The study aimed to build an educational technology for self-care for people with Chronic Kidney Disease undergoing hemodialysis from the perspective of scientific production in the area. Therefore, a qualitative, descriptive and exploratory research was carried out through an Integrative Review. Subsequently, educational technology was built from a guiding instrument. Data collection took place in January 2021 and, after applying the inclusion and exclusion criteria, a total of twenty-one articles were obtained. The results pointed to six thematic categories most prevalent with regard to the main difficulties faced by this population regarding their self-care, however, the didactic material produced integrated all the themes identified, although they did not show expressiveness from a quantitative point of view. The educational technology, the product of this study, consists of a series of five booklets that are configured in serial, distinct and interdependent by-products. This work is perceived as relevant due to the theme studied being a public health problem in Brazil and worldwide,
\end{abstract}


which impacts the quality of life of populations and requires health interventions aimed at promoting self-care. Furthermore, it is believed that the production of educational material contributes to the dissemination of health actions based on scientific evidence and, unlike other formats of academic productions, talks directly with the target audience, providing the general population with access to knowledge arising from research in the health area.

Keywords: Chronic kidney failure; Self-care; Renal dialysis.

\begin{abstract}
Resumen
El estudio tuvo como objetivo construir una tecnología educativa para el autocuidado de personas con Enfermedad Renal Crónica en hemodiálisis desde la perspectiva de la producción científica en el área. Para ello, se realizó una investigación cualitativa, descriptiva y exploratoria a través de una Revisión Integrativa. Posteriormente, la tecnología educativa se construyó a partir de un instrumento rector. La recogida de datos tuvo lugar en enero de 2021 y, tras aplicar los criterios de inclusión y exclusión, se obtuvieron un total de veintiún artículos. Los resultados apuntaron a seis categorías temáticas más prevalentes en cuanto a las principales dificultades que enfrenta esta población en cuanto al autocuidado, sin embargo, el material didáctico elaborado integró todos los temas identificados, aunque no mostraron expresividad desde un punto de vista cuantitativo. La tecnología educativa, producto de este estudio, consta de una serie de cinco folletos que se configuran en subproductos seriados, distintos e interdependientes. Este trabajo se percibe como relevante debido a que el tema estudiado es un problema de salud pública en Brasil y en el mundo, que impacta la calidad de vida de las poblaciones y requiere intervenciones de salud orientadas a promover el autocuidado. Además, se cree que la producción de material educativo contribuye a la difusión de acciones de salud basadas en la evidencia científica $\mathrm{y}$, a diferencia de otros formatos de producciones académicas, dialoga directamente con el público objetivo, brindando a la población en general acceso a los conocimientos derivados de la investigación en el área de salud.

Palabras clave: Insuficiencia renal crónica; Autocuidado; Diálisis renal.
\end{abstract}

\title{
1. Introdução
}

A Insuficiência Renal (IR) é definida como a queda da capacidade dos rins em desenvolverem suas funções de filtração, regulação hormonal e do volume de líquidos do organismo; além da regulação de outras substâncias do líquido extracelular, sendo definida como doença renal crônica quando há perda ou redução severa e irreversível da função renal por um período de, no mínimo, três meses, o que pode ser evidenciado pela presença de lesão renal bem como pela redução da taxa de filtração glomerular. O desenvolvimento da Doença Renal Crônica (DRC) ocorre de forma lenta e muitas vezes silenciosa, podendo tornar-se sintomática somente quando já houver uma perda significativa da função renal (Brasil, 2014; Stefani \& Barros, 2013).

A taxa de incidência da DRC vem aumentando da mesma forma que seu nível de morbimortalidade, o que ocorre devido ao aumento na expectativa de vida dos indivíduos, sendo esse um dos motivos pelos quais se verifica o aumento na incidência das doenças crônico-degenerativas, especialmente associadas a uma acentuada modificação nos padrões de vida (Xavier et al., 2014; Crews et al., 2019).

A DRC vem se tornando um importante problema de saúde pública e estima-se que, somente no Brasil, existam cerca de 10 milhões de pessoas acometidas pela doença (Brasil, 2014; Stefani \& Barros, 2013, Bikbov et al., 2020). Dados de 2017 apontam que, no mundo inteiro, cerca de 1,2 milhões de pessoas morreram de DRC e outras 697,5 milhões foram diagnosticadas. A taxa global de mortalidade por essa doença aumentou 41,5\% em todas as idades entre 1990 e 2017 . A DRC resultou em 35,8 milhões de agravos em incapacitantes em 2017, com a nefropatia diabética respondendo por quase um terço dessas situações (Bikbov et al., 2020).

A nível mundial, a importância global da DRC ainda não foi devidamente reconhecida. Toma-se como exemplo o Plano de Ação Global para Prevenção de Doenças Não Transmissíveis, que aborda doenças como câncer, doenças respiratórias crônicas, doenças cardiovasculares e diabetes, mas não considera a IR, mesmo após a Sociedade Internacional de Nefrologia ter demonstrado a sua relevância (Crews et al., 2019). Em contrapartida, a nível nacional a doença é devidamente reconhecida pelo Ministério da Saúde, o qual em 2014 publicou as diretrizes clínicas para o atendimento do portador de DRC 
no âmbito do SUS, bem como criou a portaria 389 de 13 de março de 2014, a qual define a organização da linha de cuidado da pessoa portadora de DRC. A DRC tem impacto significativo na morbidade e mortalidade por doenças não transmissíveis, e esta doença deve ser ativamente abordada para cumprir a meta do Objetivo de Desenvolvimento Sustentável da ONU de reduzir a mortalidade prematura por doenças não transmissíveis em um terço até 2030. (Bikbov et al., 2020)

No Brasil, no período de 2015-19 verificou-se um total de 550.917 internações por IR. No que se refere ao tratamento, houve um aumento de 32,3\% no número de centros que mantém programas de diálise no Brasil, no período compreendido entre 2009-18. Somente no ano de 2019, foram realizadas 14.579.765 sessões de hemodiálise (Brasil, 2020; Neves et al., 2020).

No que se refere à atuação do enfermeiro junto ao paciente em hemodiálise, além das competências técnicas inerentes à atuação clínica, destaca-se o desenvolvimento de atividades de educação em saúde, as quais devem ser consideradas importantes atribuições para com o paciente em hemodiálise, tanto no serviço específico para esse fim, como em unidades de internação hospitalar. As atividades educativas são capazes de empoderá-lo e transformá-lo em ator participante do seu autocuidado, cabendo ao profissional orientá-lo sobre práticas de saúde saudáveis e estimulá-lo a aderir ao tratamento, a fim de obter melhor qualidade e maior expectativa de vida (Cardoso \& Sade, 2012; Freitas et al., 2010).

Para tanto, o enfermeiro pode utilizar materiais explicativos, impressos ou digitais, que facilitem ao paciente a visualização e compreensão do processo saúde-doença e os cuidados que deve ter para melhorar sua qualidade de vida (Sampaio \& Guedes, 2012). Com esse tipo de abordagem educativa, em momentos como a alta hospitalar e a sessão de hemodiálise, é possível construir saberes para melhor gerenciar o autocuidado e, assim, tentar reduzir os elevados indicadores de internação hospitalar e mortalidade associados à DRC.

Diante disso, justifica-se a realização desse estudo com o objetivo de construir uma tecnologia educativa destinada ao autocuidado de pessoas com Doença Renal Crônica em tratamento hemodialítico sob a perspectiva da produção científica na área. Para tanto, o estudo buscou responder a questão de pesquisa: quais são as principais dificuldades enfrentadas pelos portadores de DRC em hemodiálise para o seu autocuidado?

\section{Metodologia}

Estudo de natureza qualitativa, do tipo descritivo e exploratório, para o qual utilizou-se a Revisão Integrativa (RI) como método para elaboração da tecnologia educativa, a qual constituiu-se como primeira etapa do estudo. Subsequentemente construiu-se a tecnologia educativa a partir do instrumento denominado "A Plain-Language Checklist for Creating and Reviewing Your Materials (2013)", desenvolvido pelo National Institutes of Health (NIH, 2013).

\section{Primeira etapa: Revisão Integrativa de Literatura}

A RI é um processo em que se realiza um apanhado de estudos a respeito de um tema em comum e, portanto, propiciou a associação de estudos relevantes para a fundamentação teórica e identificação dos problemas existentes no processo de autocuidado de portadores de DRC, especialmente em hemodiálise. Para a execução da RI foram executadas seis etapas subsequentes e interdependentes: 1) identificação do tema e seleção da hipótese ou questão de pesquisa; 2) estabelecimento de critérios para inclusão e exclusão de estudos ou amostragem; 3) definição das informações a serem extraídas dos estudos selecionados ou categorização dos estudos; 4) avaliação dos estudos incluídos na revisão integrativa; 5) interpretação dos resultados e 6) apresentação da revisão ou síntese do conhecimento (Mendes et al., 2008).

Realizou-se a pesquisa em janeiro de 2021, em meio eletrônico, no site Biblioteca Virtual em Saúde (BVS), especificamente nas bases de dados: Medical Literature Analysis and Retrieval System Online (MEDLINE), Bancos de Dados em Enfermagem (BDENF) e Literatura Latino- americana e do Caribe em Ciências da Saúde (LILACS). A seleção foi 
realizada por dois revisores de maneira independente como forma de sustentar a confiabilidade da coleta. Foram realizadas duas buscas distintas, na primeira utilizou-se os descritores: “doença renal crônica", "diálise renal" e "autouidado" associados ao operador boleano and, optou-se por uma segunda busca de modo a refinar a pesquisa, dessa vez sendo utilizados os termos: “diálise renal" e "autocuidado", combinados pelo operador boleano "and".

Foram definidos como critérios de inclusão para seleção: publicações disponíveis em meio eletrônico e de forma gratuita; nos idiomas português, inglês e espanhol; com texto disponível na íntegra; do período compreendido entre 2016-20; provenientes de estudos com dados primários. Como critérios de exclusão definiram-se: publicações que abordassem a DRC em pacientes pediátricos; cujo foco era a diálise peritoneal; estudos do tipo teses e dissertações; editoriais e guidelines, artigos que não tivessem a palavra autocuidado no título ou no corpo do texto, bem como, aqueles que trouxessem as dificuldades no autocuidado sob a ótica de profissionais de saúde. As publicações selecionadas que se apresentavam duplicadas foram excluídas.

A partir da leitura dos títulos e dos resumos dos artigos e, sempre que necessário, dos artigos na íntegra, verificou-se se estavam de acordo com critérios de inclusão/exclusão da RI e obteve-se um total de vinte e um artigos, desses, dezessete estavam presentes como resultado nas duas estratégias de busca e quatro estavam presentes somente na segunda. A Figura 1, disposta a seguir, ilustra a segunda estratégia de busca realizada, condensando os resultados obtidos.

Figura 1 - Fluxo de pesquisa e inclusão dos artigos da revisão integrativa com os descritores diálise renal and autocuidado Rio Grande, Rio Grande do Sul, Brasil. 2021.

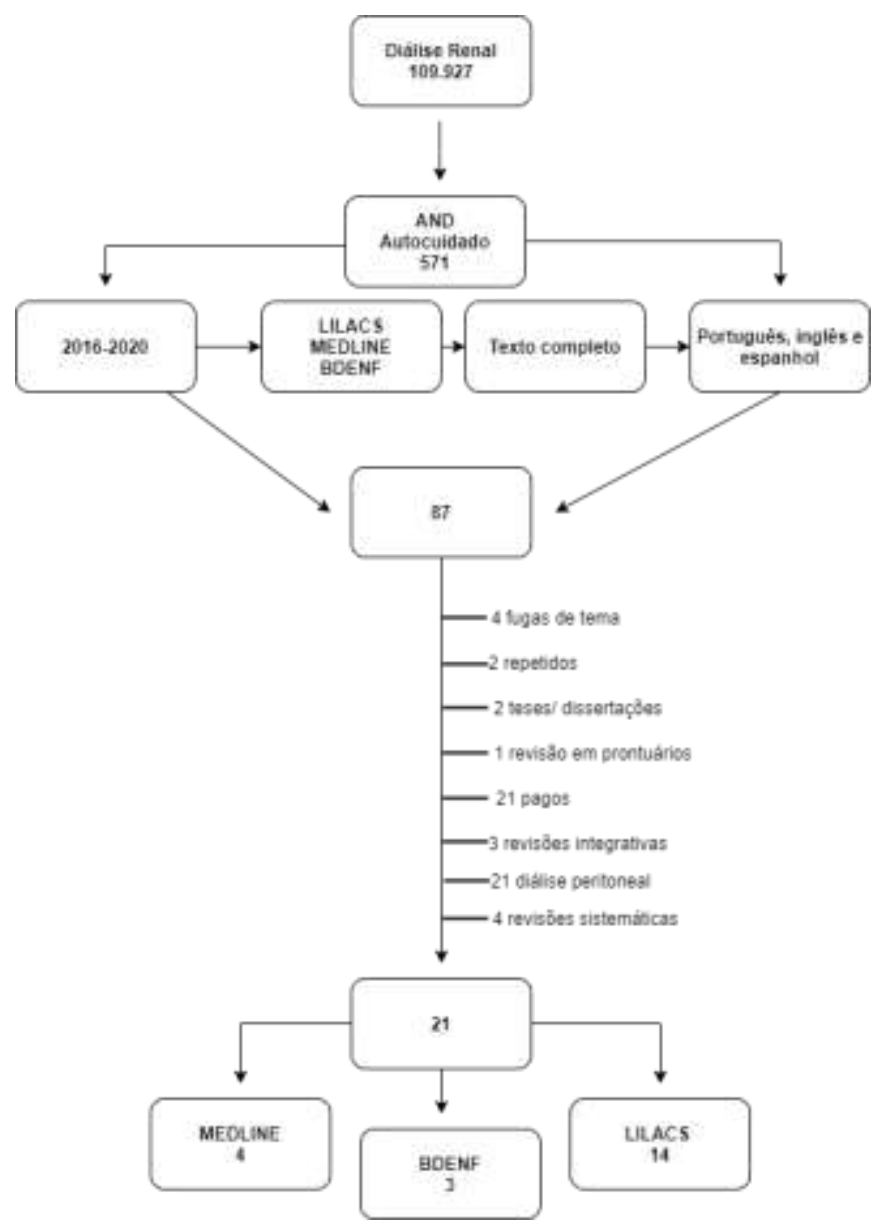

Fonte: Autores. 


\section{Segunda etapa: Construção da tecnologia educativa}

A segunda etapa do trabalho, baseou-se nos resultados alcançados na RI. Nessa etapa objetivou-se preencher as lacunas vivenciadas pelos portadores de DRC em tratamento hemodialítico, produzindo o material educativo que possa orientá-los sobre cuidados pertinentes para a compreensão da patologia; além de conhecimentos a respeito do tratamento medicamentoso, nutricional; do controle dos fatores de risco e da identificação de manifestações clínicas que indiquem necessidade de busca do serviço de saúde, de acordo com sua situação de saúde (atenção básica, serviços de nefrologia ou até mesmo emergências hospitalares).

Para tanto, o material foi construído com imagens ilustrativas, linguagem acessível e adaptada ao público-alvo de maneira a chamar atenção e despertar entusiasmo para a leitura das informações. Dessa forma, existe a garantia de tornar os resultados da pesquisa documental acessíveis ao público alvo por meio de um produto que permita a aplicabilidade na prática clínica das evidências verificadas de modo autônomo pelos pacientes.

Foi utilizado como base para a produção da tecnologia o material desenvolvido pelo National Institutes of Health (NIH, 2013), esse check-list denominado "A Plain-Language Checklist for Creating and Reviewing Your Materials (2013)", que conta com sete passos a serem seguidos para a construção de materiais educativos no âmbito da saúde.

\section{Resultados e Discussão}

Dentre os 21 artigos analisados, observou-se um maior predomínio de participantes do sexo masculino dentre os portadores de DRC (Medeiros et al., 2016; Bettoni; Ottaviani; Orlandi, 2017; Clementino et al., 2018; Santos; Lira; Fernandes, 2019; Karbalaelfar et al., 2016; Yang et al., 2019; Pennafort et al., 2019). Em 2017 a incidência global por idade de diálise e transplante foi 1,47 vezes maior entre os homens do que entre mulheres e a taxa global de mortalidade por DRC por idade foi 1,39 vezes maior entre os homens do que entre as mulheres (Bikbov et al., 2020).

A idade dos participantes desses estudos variou entre 18 a 87 anos, sendo a média de idade presente nos estudos de 53,2 anos. Corroborando com estes resultados, outros estudos também apresentam o perfil mais recorrente do doente renal crônico sendo do sexo masculino, com média de idade variando entre 50 e 58 anos (Dantas et al., 2019; Lins et al., 2018; Hartwig; Junior; Ignotti, 2018; Alves et al., 2018; Piccin et al., 2018). No que tange à escolaridade, a maioria das publicações não abordou essa informação e, dentre as que trouxeram, houve maior predominância de participantes com ensino fundamental incompleto (Bettoni; Ottaviani; Orlandi, 2017; Clementino et al., 2018) ou ensino fundamental completo (Santos; Lira; Fernandes, 2019; Mendonça et al., 2020). A seguir, o Quadro 1 apresenta as categorias encontradas ao longo da RI que dizem respeito às dificuldades encontradas pelos portadores de DRC em hemodiálise para o seu autocuidado e sua correspondente frequência nas publicações da amostragem. 
Quadro 1: Categorias temáticas e frequência verificada em relação às dificuldades encontradas por portadores de DRC. Rio Grande, Rio Grande do Sul, Brasil, 2021.

\begin{tabular}{|l|c|}
\hline Categoria & Frequência \\
\hline Cuidados com a fistula arteriovenosa & 11 \\
\hline Aspectos psicológicos & 9 \\
\hline Alimentação & 8 \\
\hline Autocuidado & 7 \\
\hline Déficit de conhecimento sobre a DRC e atitudes de autocuidado & 6 \\
\hline Consumo de líquidos & 4 \\
\hline Hemodiálise & 4 \\
\hline Família relacionada ao autocuidado & 3 \\
\hline Sexualidade & 3 \\
\hline Religiosidade & 2 \\
\hline Atividade física & 2 \\
\hline Terapia medicamentosa & 1 \\
\hline Letramento funcional em saúde & 4 \\
\hline
\end{tabular}

Fonte: Autores.

Para essa publicação, serão apresentadas e discutidas as temáticas prevalentes identificadas com frequência igual ou acima de 5 devido à necessidade de síntese dos resultados e análise realizada. No entanto, o material didático produzido a partir da RI foi construído considerando todas as temáticas verificadas no estudo, ainda que não tenham apresentado expressividade do ponto de vista quantitativo.

Dentre as dificuldades enfrentadas pelos portadores de DRC relacionadas à fístula arteriovenosa (FAV), emergiram o efeito doloroso das punções para realização da hemodiálise; as restrições físicas devido à presença da fístula; os cuidados com a FAV, além das possíveis complicações com a FAV e a sua relação com algumas doenças. Ainda, somam-se aos dificultadores para o autocuidado relacionado à FAV, o déficit de conhecimento em relação à sua função e confecção; a falta de orientações acerca de cuidados necessários no processo de maturação da fístula; assim como, de cuidados domiciliares para a FAV em situações de baixo fluxo, sangramentos, hematomas e sinais de infecção (Santos et al., 2017; Salimena et al., 2016; Araújo et al., 2016; Medeiros et al., 2016; Clementino et al., 2018; Matias et al., 2020; Lucca et al., 2020; Neto et al., 2016; Mendonça et al., 2020; Yang et al., 2019; Pennafort et al., 2019).

As pessoas podem apresentar déficit de conhecimento a respeito da FAV, visto que, por vezes, compreendem a mesma como algo externo à elas, ou seja, como algo que foi instalado, sendo relacionado como algo anormal, embora tenham compreensão de que a fístula é responsável pela manutenção da sua vida (Neto et al., 2016).

O conhecimento que a maioria dos portadores de DRC tem são insuficientes para o desenvolvimento de uma boa prática de autocuidado (Neto et al., 2016; Mendonça et al., 2020). Os resultados da pesquisa de Yang et al. (2019) ratificam os achados anteriores, pois demonstraram que somente $30,1 \%$ dos participantes estavam com níveis altos de autocuidado, o que propõe que os pacientes tem algum conhecimento a respeito dos cuidados necessários com a FAV, porém, são insuficientes. 
Deste modo, devem ser realizadas atividades educativas que envolvam os cuidados com a FAV, nas quais se possa utilizar uma linguagem clara e compreensível, a fim de contribuir com a qualidade de vida dos pacientes (Salimena et al., 2016). Alguns cuidados necessários com a fístula, são: verificar, a partir da palpação do frêmito, o funcionamento do acesso; observar a presença de sinais e sintomas de infecção, os quais devem ser comunicados imediatamente para a equipe de saúde; realizar a higiene do membro; não verificar a pressão arterial, assim como, não realizar punções venosas no braço da fístula; não dormir sobre o membro e evitar qualquer atividade que possa causar compressão (Neto et al., 2016; Pennafort et al., 2019; Matias et al., 2020).

Evidências indicam uma grande incidência de pacientes que mantém práticas insuficientes de autocuidado, além de destacar que esses cuidados com a FAV previnem o surgimento de complicações, como: trombose, estenose, baixo fluxo, isquemia da mão, aneurisma, pseudoaneurisma, infecções, hematomas, sucção excessiva no influxo (arterial) e pressão venosa alta (Mota; Almeida; Santos, 2018). Em relação ao período de maturação evidencia-se a necessidade de que sejam tomados cuidados específicos para que não haja interrupção do fluxo sanguíneo no local. Dentre esses cuidados se podem citar: suspender o membro nos primeiros dias; manter o curativo limpo e seco, evitando curativos oclusivos que possam interromper o fluxo sanguíneo; verificar a presença de pulso e frêmito; evitar hipotensão (Neto et al., 2016; Pennafort et al., 2019). Os cuidados tem por objetivo assegurar um maior tempo de duração da FAV e prevenir uma dificuldade também destacada na RI, no que diz respeito à necessidade de submissão à diversos procedimentos para confecção de novas fístulas (Santos et al., 2017; Santana, Nobre e Luz, 2019).

Outro fator que dificulta o autocuidado do doente renal crônico em hemodiálise é a alimentação. Sobre a alimentação, foram abordados: quais os alimentos podem ou não ser consumidos e em que quantidade; tempo de cozimento dos alimentos; cuidados com o consumo de sal e substituição por temperos naturais (Santos et al., 2017; Salimena et al., 2016; Araújo et al., 2016; Xavier et al., 2017; Medeiros et al., 2016; Santana et al., 2020; Lucca et al., 2020; Griva et al., 2019).

A alimentação, para algumas pessoas, pode ser o aspecto mais difícil de ser enfrentado, é comum que apresentem resistência para mudar hábitos e, por vezes, desrespeitem a dieta na véspera da hemodiálise por acreditar que essa irá restabelecer as funções normais do organismo (Santana et al., 2020; Perusso et al., 2019). As dificuldades podem estar relacionadas sob o ponto de vista individual como a situação econômica do paciente, caráter rigoroso da dieta, paladar, nível educacional, status social, comportamento e crenças religiosas (Santana et al., 2020, Lins et al., 2018).

O próprio autocuidado apresentou uma abordagem múltipla de acordo com as seguintes temáticas: relação entre maior conhecimento, maior atitude, prática e autocuidado; melhores níveis de autocuidado na população mais madura e vinculação desse melhor autocuidado com redução dos níveis de fósforo sérico e potássio (Karbalaeifar et al., 2016; Santana et al., 2020; Lucca et al., 2020; Kim; Kim; Ryu, 2019; Neto et al., 2016; Yang et al., 2019; Clementino et al., 2018).

Karbaleifar et al. (2016) concluíram que os níveis de autocuidado foram menores nos pacientes com mais de 10 anos de diagnóstico; os níveis de prática e de autocuidado foram maiores entre aqueles que utilizavam medicação para tratamento do diabetes; pacientes com histórico familiar de diabetes mellitus apresentaram escores menores de autocuidado. Maior escolaridade esteve relacionada com maior conhecimento, prática e autocuidado. E, entre os fatores de risco para o desenvolvimento de DRC, o tabagismo e a hipertensão estiveram relacionados com menores níveis de autocuidado. Esses autores perceberam uma relevante relação entre o conhecimento e a prática do paciente e o seu autocuidado, inferindo que, ao orientar o paciente, aumenta-se a sua conscientização a respeito da doença o que, consequentemente, melhora a sua atitude e a sua prática em relação à si e à doença, traduzindo-se, portanto, em melhora no autocuidado.

Os estudos abordaram em parte as questões psicológicas relacionadas com a FAV e, nesse sentido surgiram: alteração da imagem corporal devido à FAV ou ao cateter duplo-lúmen; baixa autoestima e sensação de imperfeição; sentimento de rejeição e discriminação, repulsa ou raiva da FAV, tristeza, impotência, vergonha e sofrimento (Santos et al., 
2017; Silva et al., 2018; Neto et al., 2016). As publicações ainda elencaram as questões psicológicas associadas à hemodiálise, de forma geral. Acerca disso, foram abordadas as repercussões negativas acarretadas pela dependência das máquinas de hemodiálise e da necessidade de ajuda dos familiares. O fato do indivíduo não poder trabalhar gera sentimentos de inadequação, desamparo e ociosidade e, também, ocasiona medo de se tornar um ônus aos seus familiares. Não obstante, sintomas de depressão são comuns aos pacientes em hemodiálise, além de humor deprimido persistente, autoimagem prejudicada, sentimentos pessimistas, baixa autoestima, dificuldade de aceitação, preocupação, insegurança e desesperança com o futuro; medo, revolta e ansiedade também são comuns nessas pessoas (Santos et al., 2017; Santos et al., 2016; Araújo et al., 2016; Xavier et al., 2017; Griva et al., 2019; Neto et al., 2016).

A formação de vínculo do profissional de saúde com o doente renal crônico em hemodiálise favorece a diminuição da sintomatologia psicológica visto que recebe apoio para encarar a doença e o próprio tratamento (Santos et al., 2017). Para Salimena et al. (2016), os profissionais de saúde devem estar prontos para apoiar os pacientes em início de terapia hemodialítica visto que, durante a adaptação, surgem sentimentos como revolta e ansiedade acarretados pelas mudanças fisiológicas e psicológicas próprias do momento.

O déficit de conhecimentos sobre a DRC foram citados em seis artigos e, neste sentido, foi relatada falta de conhecimento no que diz respeito à patologia em si, sinais e sintomas de complicações, intercorrências possíveis na DRC, relação da hipertensão e do diabetes com a doença, os agravos da DRC; bem como, a respeito da fadiga relacionada com a doença; as formas de prevenção da sua progressão e; aos efeitos da DRC na qualidade de vida (Salimena et al., 2016; Araújo et al., 2016; Medeiros et al., 2016; Bettoni; Ottaviani; Orlandi, 2017; Luca et al., 2020; Santana et al.;2020; Neto et al; 2016; Yang et al.; 2019).

Os pacientes podem, no início do tratamento, apresentar dificuldades no autocuidado devido à falta de conhecimento sobre a doença, tratamento e restrições necessárias impactanto negativamente na adoção de práticas de autocuidado, dificultando a compreensão das orientações, o que pode levar ao abandono do tratamento (Maciel et al., 2015; Lucca et al., 2020). Dessa forma, as atividades educativas surgem como uma possibilidade de promoção de saúde e buscam empoderar o paciente para que possa ter suas práticas voltadas ao autocuidado.

O consumo de líquidos foi apresentado como dificultador do autocuidado, sendo a quantidade limitada de líquidos permitida diariamente o principal desafio (Santos et al., 2017; Salimena et al., 2016; Araújo et al., 2016; Xavier et al., 2017; Santana et al., 2020). Santos et al. (2017) e Araújo et al. (2016) somente citam a necessidade de mudança de hábitos no consumo de líquidos por doentes renais crônicos em hemodiálise, sugerindo que evite-o em excesso. Para tanto, evidenciam a importância de orientações para o autocuidado. Salimena et al. (2016) ratificam a necessidade de informar o paciente a respeito da ingesta de líquidos e reconhecem que a limitação do consumo de água é um estressor para os indivíduos.

No estudo de Xavier et al. (2017) o consumo de líquidos foi considerado um item de baixo controle. Já Santana et al. (2020) concluíram que o consumo de líquidos, juntamente com a alimentação, foi considerado uma das principais demandas de autocuidado, sendo que vários participantes reconheceram a importância deste cuidado, embora tivessem dificuldades para realizá-lo. Devido à essas dificuldades, os participantes do estudo declararam ingerir mais água do que deveriam, priorizando este quesito em comparação à alimentação.

A segunda etapa do presente estudo consistiu, no desenvolvimento da tecnologia educativa, a qual foi baseada nas categorias encontradas na RI, essas, evidenciaram as lacunas destacadas pelos portadores de DRC em hemodiálise para o seu autocuidado. A tecnologia foi construída em formato de cartilha e foi dividida em cinco subcapítulos que apresentam complementaridade, apesar de possibilitarem a leitura individual e independente. O primeiro discorre sobre a doença renal crônica; o segundo, apresenta a hemodiálise; o terceiro refere-se aos acessos para hemodiálise; o quarto aborda os cuidados 
com a alimentação na doença renal crônica e a hemodiálise e o último, os cuidados com a atividade física, o uso de medicamentos, a rotina de exames e a saúde mental na doença renal crônica e a hemodiálise.

Utilizou-se o check-list intitulado "A Plain-Language Checklist for Reviewing Your Document" da NIH de 2013 como embasamento para o desenvolvimento da tecnologia educativa; levando-se em consideração os sete aspectos citados no referido check-list. O primeiro deles, diz respeito à apropriação do público alvo, o que ocorreu através da RI, a partir do que foi possível traçar do perfil sociodemográfico dos pacientes renais crônicos em hemodiálise. A seguir, o segundo aspecto diz respeito ao conteúdo, se o mesmo vai ao encontro ao interesse do leitor. Nesse sentido, a RI também se mostrou importante, visto que destacou os principais pontos a serem discutidos na cartilha. (NIH, 2013).

O terceiro e quarto aspectos dizem respeito ao método utilizado para "conversar" com o leitor, sugerindo-se, então, a utilização de primeira e segunda pessoa para a voz do narrador, de forma que a leitura torne-se mais conversacional e fluida. Além disso, se recomenda no referencial a não utilização de terminologias técnicas e, sim, o uso de uma linguagem clara e objetiva, com parágrafos de no máximo sete frases e de dez a vinte palavras, de forma que o material fique atrativo.

A quinta etapa diz respeito às ilustrações do material construído, as quais devem se relacionar com o leitor. Para tanto, utilizaram-se figuras que ilustrassem as orientações e informações oferecidas visto que, de acordo com a RI, o perfil do doente renal crônico em hemodiálise está relacionado à uma baixa escolaridade (ensino fundamental incompleto). Visto isso, optou-se pela utilização de ilustrações e linguagem de fácil compreensão (NIH, 2013).

A próxima etapa diz respeito à escrita em voz ativa, o que leva à ideia de ação de modo a motivar e estimular o leitor. Nesse sentido, buscou-se construir três personagens, os quais interagem entre si a fim de que o leitor se sinta representado em suas principais dúvidas para o autocuidado, explicando-se de forma clara e objetiva as informações contidas nas categorias da RI. O sétimo aspecto está relacionado aos títulos dos capítulos, os quais devem ser claros e objetivos, de forma que o leitor se situe sobre o assunto abordado (NIH, 2013).

A cartilha foi avaliada pelos membros da banca de TCC para o qual esse trabalho foi utilizado, a banca era composta por expertises nas áreas de design, de doença renal crônica e hemodiálise e de doenças crônicas. A cartilha será posteriormente validada diretamente com os portadores de DRC em hemodiálise.

A série "Vivendo com a Doença Renal Crônica e a Hemodiálise" é composta por cinco cartilhas seriadas e interdependentes. A cartilha "Conhecendo a Doença Renal Crônica (DRC)" discorre sobre a função renal, fisiopatologia da DRC, fatores de risco associados, a relação com a Hipertensão Arterial Sistêmica e Diabetes Mellitus assim como, a identificação de manifestações clínicas e possíveis complicações da doença.

A cartilha "A Hemodiálise" apresenta o tratamento, trazendo informações relevantes sobre o procedimento, efeitos esperados, possíveis sinais e sintomas durante o processo e acessos vasculares para hemodiálise (catéter duplo lúmen e a fístula arteriovenosa). A cartilha "Cuidados com os acessos para hemodiálise" aborda cuidados específicos com o cateter duplolúmen e com a FAV, cuidados necessários durante o tempo de maturação da FAV, relação da pressão arterial com a FAV, complicações relacionadas com a FAV e condutas a serem adotadas diante de cada possível complicação.

A cartilha "Cuidados com a alimentação na DRC e hemodiálise" traz uma série de informações que inicialmente abordam a importância de manter os cuidados com a ingesta alimentar para a pessoa que vive com a DRC. Posteriormente destaca alimentos que devem ser evitados, a quantidade diária recomendada de ingestão de fósforo e sódio além de, sinais e sintomas associados a elevados níveis de fósforo, ureia, potássio, sódio assim como, excesso de consumo de proteínas e líquidos. São apontadas ainda alternativas e cuidados para o consumo adequado dos alimentos, quantidades recomendadas para o consumo de acordo com o teor de potássio, restrições em relação ao consumo de sódio e uso de temperos substitutivos. O material também contempla um "Cardápio semanal” para ser impresso e preenchido pela própria pessoa com DRC ou familiar/cuidador de acordo com as próprias preferências alimentares - que podem ser melhor discutidas e ajustadas com 
um(a) nutricionista. Ademais a cartilha trata do controle de consumo de líquidos e apresenta a conversão de unidades de medida para o cálculo diário, alternativas que facilitam esse controle. Por fim, é apresentado brevemente um aplicativo de celular que funciona como uma ferramenta digital facilitadora com alguns recursos importantes como controle de volume de líquidos, peso corporal, exames, medicações e tabela nutricional.

E por último, a cartilha "Cuidados com a atividade física, o uso de medicamentos, a rotina de exames e a saúde mental na Doença Renal Crônica e a Hemodiálise” apresenta possibilidades de atividades físicas permitidas e tempo necessário para cada uma, periodicidade de exames laboratoriais, importância do uso correto das medicações, dicas para organização da administração das medicações e também, um quadro organizador para ser impresso e preenchido pela própria pessoa com DRC ou familiar/cuidador de acordo com a terapêutica medicamentosa instituída. Essa cartilha ainda aborda a necessidade de identificar a percepção pessoal de humor a partir da aplicação de uma escala visual e possível encaminhamento a ser avaliado e conduzido por um profissional de saúde de acordo com o resultado da aplicação da escala.

A seguir serão apresentados recortes da tecnologia educativa, a qual se originou a partir dos resultados encontrados na RI e se encontra disponível para download de forma gratuita no site da Escola de Enfermagem da FURG, endereço eletrônico: https://eenf.furg.br

\section{Figura 1.}
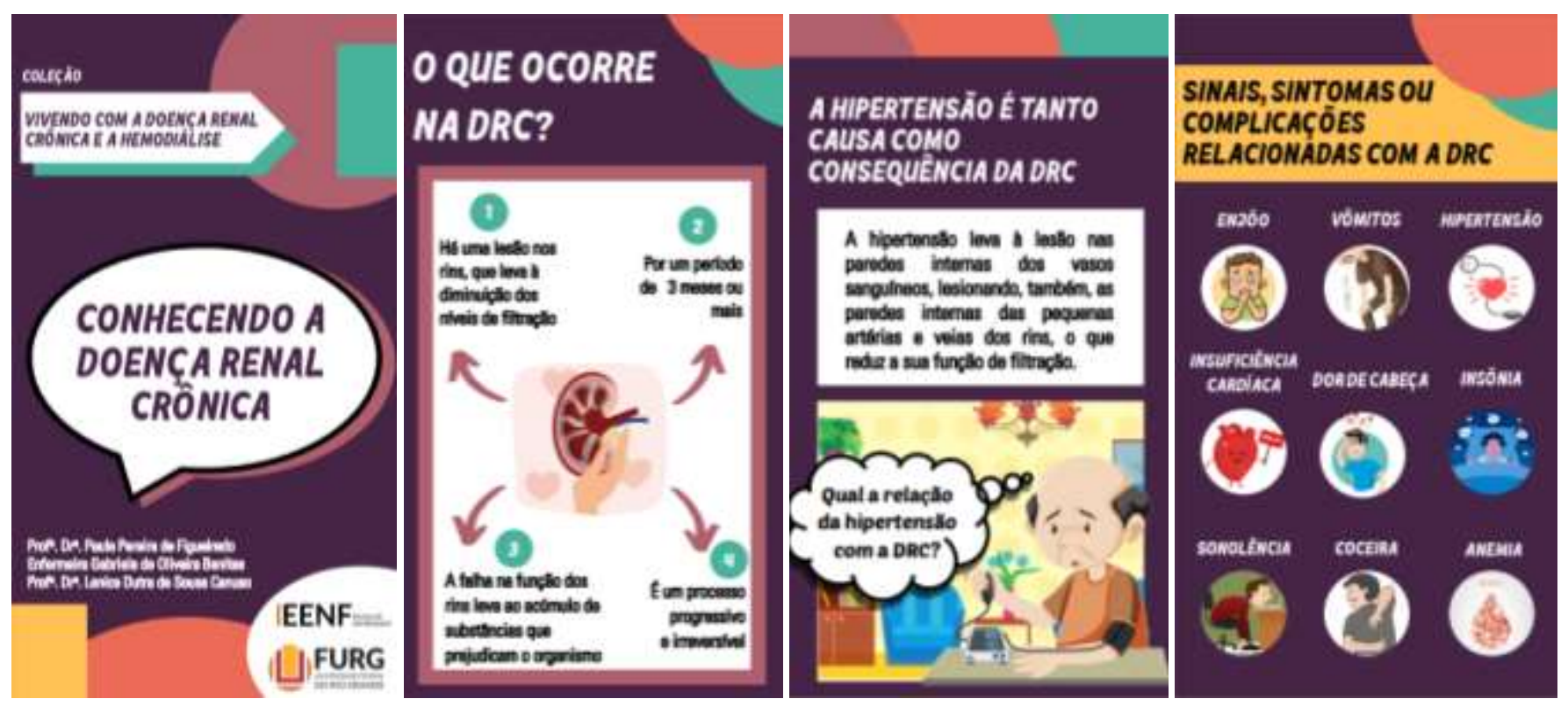
Research, Society and Development, v. 11, n. 2, e14711222269, 2022

(CC BY 4.0) | ISSN 2525-3409 | DOI: http://dx.doi.org/10.33448/rsd-v11i1.22269
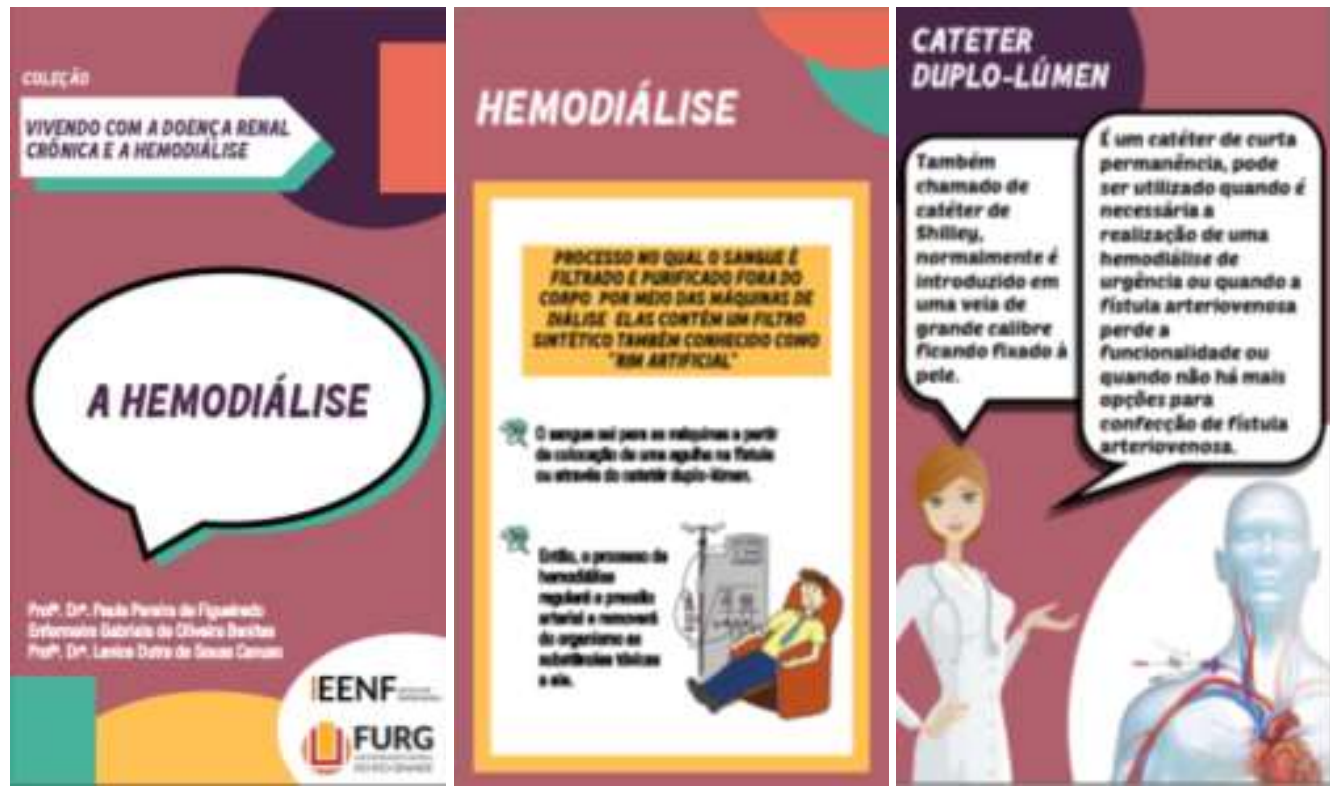

\section{FISTULA \\ ARTERIOVENOSA}

f um acesso vascular feito a partir da unilas de uma arteria e uma veia. f feito por meio de uma cirurgia simples, precisando apenas de anestesia local.

(19)
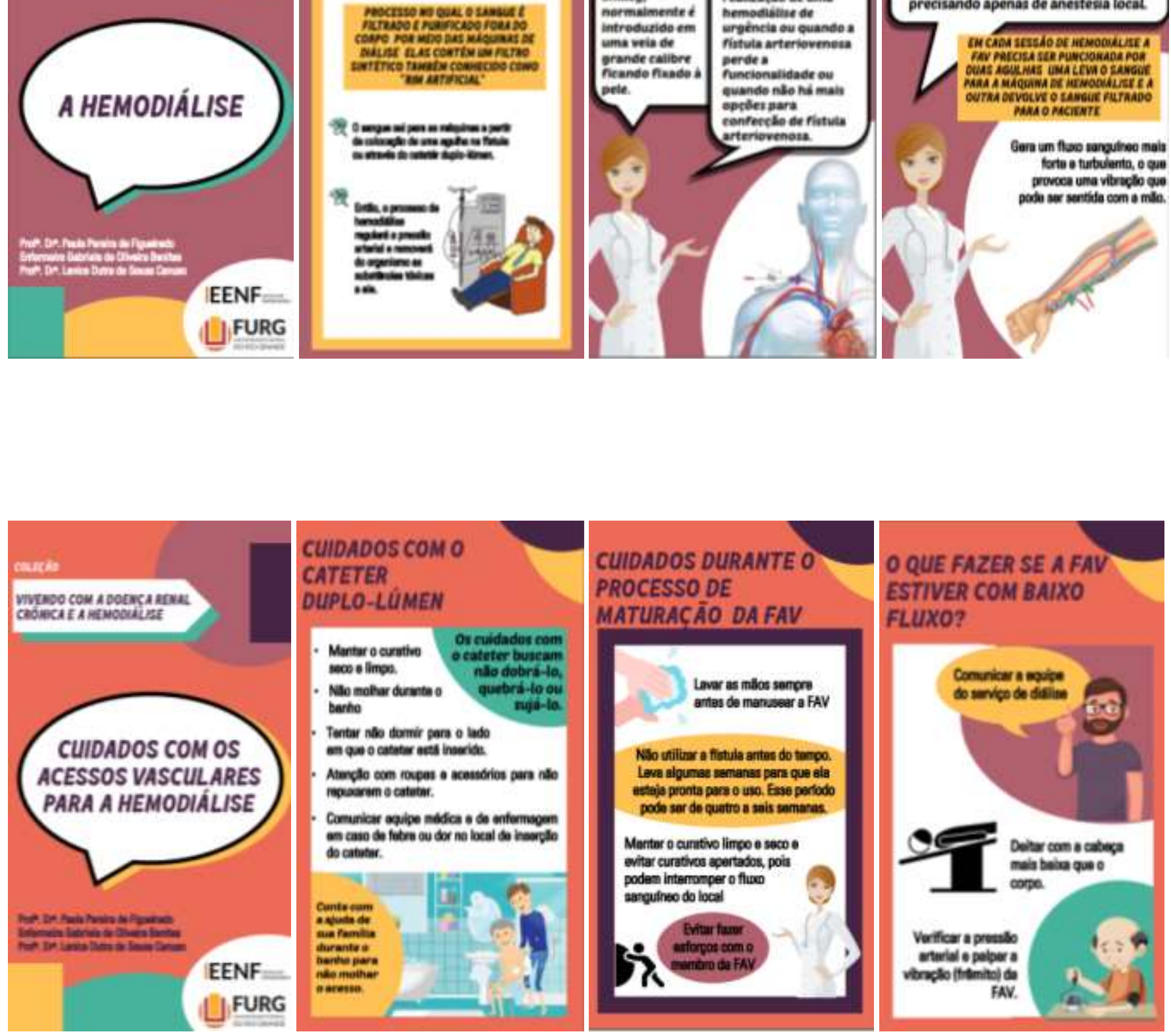

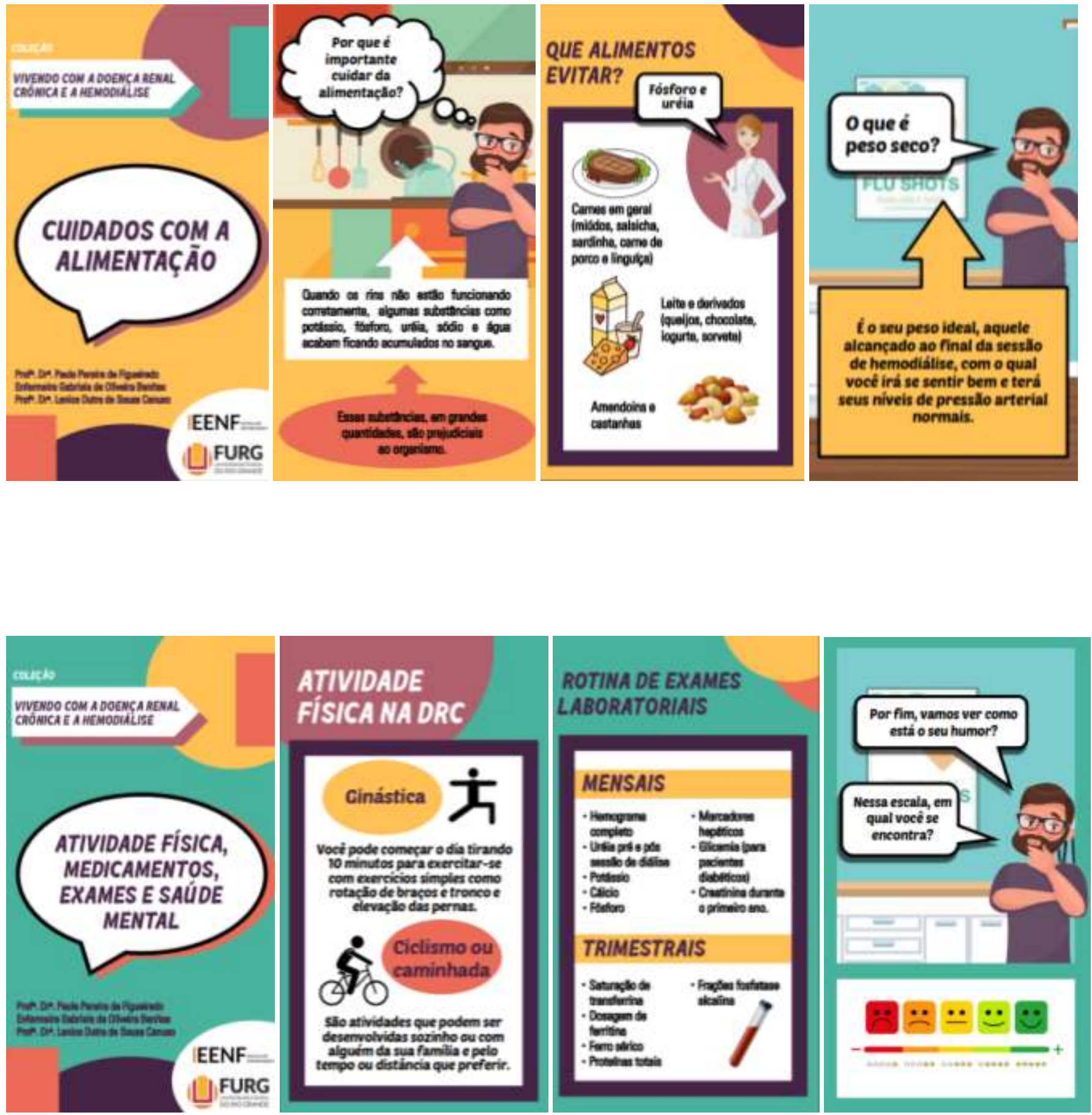

Fonte: Autores.

\section{Considerações Finais}

O estudo identificou seis categorias temáticas mais prevalentes no que diz respeito as principais dificuldades enfrentadas pelos portadores de DRC em hemodiálise para o seu autocuidado a partir da produção científica na área. Verificouse portanto, que os cuidados com a fístula arteriovenosa, a alimentação, o autocuidado, os aspectos psicológicos, o déficit de conhecimento sobre a DRC e o consumo de líquidos são aspectos identificados com mais frequencia como dificultadores para o autocuidado do paciente com DRC. Contudo a escassez de publicações na área do estudo indica que essa é uma temática ainda pouco abordada e, portanto, o material didático produzido a partir da RI foi construído considerando não só as categorias 
temáticas mais frequentemente verificadas, mas integrou todas as temáticas identificadas, ainda que não tenham apresentado expressividade do ponto de vista quantitativo.

Observou-se que as publicações que constituíram a amostra deste estudo apresentam resultados de pesquisas que destacam as dificuldades encontradas pelos pacientes renais crônicos em hemodiálise. Contudo, se tratam de produções que não apontam a resolutividade em resposta a essa dificuldades, o que fragiliza uma sustentação teórica baseada em evidências científicas para a aplicabilidade na prática clínica em Enfermagem.

Percebe-se esse trabalho como relevante devido à temática estudada apresentar-se como um problema de saúde pública no Brasil e no mundo, que impacta na qualidade de vida das populações e que requer intervenções em saúde com vistas à promoção do autocuidado para pessoas que vivem com a DRC. Ademais, acredita-se que a produção do material educativo contribui para a divulgação de ações em saúde pautadas em evidências científicas e, diferente de outros formatos de produções acadêmicas, conversa diretamente com o público-alvo, oportunizando à população em geral o acesso ao conhecimento advindo de pesquisas na área da saúde.

A tecnologia educativa construída nesse estudo, não abordou nenhuma área específica dentro da DRC e, por esse motivo, tornou-se um material longo visto que abordou diversas temáticas. Deste modo, optou-se por fragmentar o material produzido em uma série de cinco cartilhas que se configuram em subprodutos seriados, distintos e interdependentes. Outros autores que também desenvolveram esse tipo de material o fizeram sobre determinados assuntos a respeito da doença, de forma que seus materiais ficaram menos extensos.

Almeja-se que a tecnologia educativa, principal produto deste estudo, configure-se em um instrumento de orientação para o autocuidado de pacientes renais crônicos e que posteriormente seja testada, validada e aprovada por experts na temática e pelos próprios pacientes renais crônicos em situação de hemodiálise, estando sujeito e aberto a modificações e sugestões ao longo de todo processo.

Sugere-se que outros trabalhos venham a realizar pesquisas no âmbito do autocuidado, bem como a continuidade do estudo das fragilidades encontradas pelos portadores de DRC em situação de hemodiálise para seu autocuidado. Além disso, é de suma importância a continuidade desse estudo para que a cartilha seja apresentada aos doentes renais crônicos e validada pelos mesmos.

\section{Referências}

Alves, K. B. et al. Existe associação entre qualidade de vida e adesão à farmacoterapia em pacientes com doença renal crônica em hemodiálise? . Einstein, [s. l.], 2018.

Araújo, J. B. de et al. Cotidiano de pacientes renais crônicos submetidos à hemodialise: expectativas, modificações e relações sociais. Revista Online de

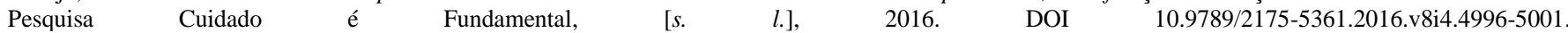
http://www.seer.unirio.br/index.php/cuidadofundamental/article/view/4404.

Bettoni, L. C.; Ottaviani, A. C.; Orlandi, F. S. Associação entre o autocuidado e a qualidade de vida de pacientes com doença renal crônica. Revista Eletrônica de Enfermagem, v. 19, 28 nov. 2017.

Brasil, Ministério da Saúde, Secretaria de Atenção à Saúde, \& Departamento de Atenção Especializada e Temática. (2014). Diretrizes Clínicas para o Cuidado ao paciente com Doença Renal Crônica - DRC no Sistema Único de Saúde.

Brasil, Ministério da Saúde, DataSus (2020). http://tabnet.datasus.gov.br/cgi/tabcgi.exe?sih/cnv/nirs.def

Bikbov, Boris et al. Global, regional, and national burden of chronic kidney disease, 1990-2017: a systematic analysis for the Global Burden of Disease Study 2017. Lancet, 2020. DOI 10.1016 / S0140-6736 (20) 30045-3. https://www.thelancet.com/action/showPdf?pii=S0140-6736\%2820\%2930045-3

Cardoso, L. B., \& Sade, P. M. C. (2012). O enfermeiro frente ao processo de resiliência do paciente em tratamento hemodialítico. Revista Eletrônica da Faculdade Evangélica do Paraná.

Clementino, Daniella Caldas et al. Pacientes em hemodiálise: importância do autocuidado com a fístula arteriovenosa. Revista de Enfermagem UFPE Online, [s. l.], 2018. DOI 10.5205/1981-8693-v12i7a234970p1841-1852-2018. https://pesquisa.bvsalud.org/portal/resource/pt/biblio-986528. 
Couto, Dálete et al. Assistência de enfermagem ao paciente estomizado baseado na teoria de dorothea orem. Brazilian Journal of Surgery and Clinical Research, [S. l.], p. 55-58, 15 fev. 2018.

Crews, D. C., Bello, A. K., \& Saadi, G. (2019). Editorial do Dia Mundial do Rim 2019 - Impacto, acesso e disparidades na doença renal. Kidney International, 95, $242-248$.

Dantas, Lianna G. G. et al. Non-adherence to Haemodialysis, Interdialytic weight gain and cardiovascular mortality: a cohort study. BMC Nephrology, [s. l.], 2019 .

Faria, Nayara Vieira de; TEIXEIRA, Cecilma Miranda de Sousa; NUNES, Simony Fabíola Lopes. Conhecimento dos usuários do programa hiperdia sobre a doença renal crônica. Journal of Managelment \& Primary Health Care, [S. l.], p. 4-9, 8 mar. 2014.

Freitas, T. F. d., Oliveira, E. R. V. d., Vellinho, L. P. B., Rocha, P. N. M. d., Monteiro, L. A. H., \& Souza, S. R. d. (2010). Enfermagem e ações educativas em portadores de insuficiência renal crônica. Revista de pesquisa: cuidado é fundamental online. http://www.scielo.br/pdf/reeusp/v41n4/12.pdf

Galvão, Maria Teresa dos Reis Lopes Silveira; Janeiro, José Manuel da Silva Vilelas. O autocuidado em enfermagem: autogestão, automonitorização e gestão sintomática como conceitos relacionados. Revista Mineira de Enfermagem, [s. l.], 2013.

Garcia-Canton, C., Rodenas, A., Lopez-Aperador, C., Rivero, Y., Anton, G., Monzon, T., Diaz, N., Vega, N., Loro, J. F., Santana, A., \& Esparza, N. (2019). Frailty in hemodialysis and prediction of poor short-term outcome: mortality, hospitalization and visits to hospital emergency services. Journal Renal Failure.

Griva, K et al. THE COMBINED diabetes and renal control trial (C-DIRECT) - A feasibility randomised controlled trial to evaluate outcomes in multi-morbid patients with diabetes and on dialysis using a mixed methods approach. BMC Nephrology, [s. l.], 2019. DOI 10.1186/s12882-018-1183-z. https://www.ncbi.nlm.nih.gov/pmc/articles/PMC6318946/.

Hartwig, S. V.; Junior, A. L. de S.; Ignotti, E. Medicamentos para hipertensão de pacientes em hemodiálise em Cáceres - Mato Grosso, Brasil. O mundo da saúde, [s.l.], 2018.

Karbalaeifar, R. et al. Evaluating the effect of knowledge, attitude and practice on self-management in patients with type 2 diabetes. Journal of Diabetes Research, [S. l.], p. 1-7, 19 maio 2016. DOI 10.1155/2016/3730875. https://www.hindawi.com/journals/jdr/2016/3730875/.

Lima, M. A. de et al. Educação em saúde para pacientes em hemodiálise. Revista de Enfermagem UFPE Online, [s. l.], 2014.

Lins, S. M. de S. B. et al. Adesão de portadores de doença renal crônica em hemodiálise ao tratamento estabelecido. Revista Acta Paulista de Enfermagem, [s. l.], 2018.

Lucca, D. C. et al. Jogo das Atitudes: gerontotecnologia educacional para idosos em tratamento hemodialítico. Revista Brasileira de Enfermagem, [s. l.], 27 abr. 2020. DOI 10.1590/0034-7167-2018-0694. https://www.scielo.br/scielo.php?pid=S0034-71672020001500165\&script=sci_abstract\&tlng=pt.

Maciel, C. de G. et al. Adesão ao tratamento hemodialítico: percepção dos pacientes renais crônicos. Cogitare Enfermagem, [s. l.], 2015.

Matias, D. M. de M. et al. Cuidado individual domiciliar de pacientes com fístula arteriovenosa. Revista de Enfermagem UFPE Online, [s. l.], 2020. DOI 10.5205/1981-8963.2020.244317. Disponível em: cuidado individual domiciliar de pacientes com fístula arteriovenosa. Acesso em: 29 jan. 2021.

Medeiros, J. R. R. de et al. Validação de tecnologia educativa para cuidado em hemodiálise. Revista de Enfermagem UFPE Online, [s. l.], 2016. DOI 10.52205/reuol.9881-87554-1-EDSM1011201614. Disponível em: https://periodicos.ufpe.br/revistas/revistaenfermagem/article/view/11474. Acesso em: 29 jan. 2021.

Mendes, K. D. S., Silveira, R. C. d. C. P., \& Galvão, C. M. (2008). Revisão integrativa: método de pesquisa para a incorporação de evidências na saúde e na enfermagem. Texto e Contexto Enfermagem, 17(04), 758-764.

Mendonça, A. E. O. de et al. Autocuidado do paciente renal com a fístula arteriovenosa. Revista Enfermagem em Foco, [s. l.], p. 181-187, 2020.

Mota, Y. K. P.; Almeida, M. M.; Santos, M. S. The knowledge of the chronic renal patient about the arteriovenosa fistula and its implications in selfcare. Revista Ciência \& Saberes, [s. l.], 27 set. 2018.

Neto, J. M. R.; Rocha, E. R. dos S.; Almeida, A. R. M. de; Nóbrega, M. M. L.a da. Fístula arteriovenosa na perspectiva de pacientes renais crônicos. Revista Enfermagem em Foco, [s. l.], 2016.

Mulder, B. A.,Veldhuisen, D. J. V., Crijn, H. J. G. M., Tijssen, J. G. P., Hillege, H. L., Alings, M., Rienstra, M., Groenveld, H. F., Berg, M. P. D. B., \& Gelfer, I. C. V. National Institutes of Health- NIH, (2013). A Plain-Language Checklist for Reviewing Your Materials. https://www.nih.gov/sites/default/files/institutes/plain-language/nih-plain-language-checklist.pdf

Noleto, L. C., Fonsêca, A. C. d., Luz, M. H. B. A., Batista, O. M. A., \& Pereira, A. F. M. (2015). O papel dos profissionais de enfermagem no cuidado ao paciente em tratamento hemodialítico: revisão integrativa. Revista de Enfermagem UFPE.

Pennafort, V. dos S. P.; Lobo, F. L. M.; Barbosa, T. O.; Pontes, F. G. Tecnologia educacional para orientação de idosos nos cuidados com a fístula arteriovenosa. Enfermagem em Foco, [s. l.], p. 79-84, 2019.

Perusso, F. K. G. et al. Alimentação e hábitos de vida na doença renal crônica. Revista Caderno de Medicina, [s. l.], 2019.

Piccin, C. et al. Perfil sociodemográfico e clínico de pacientes renais crônicos em hemodiálise. Revista de Enfermagem, [s. l.], 2018.

Pivatto, D. R., \& Abreu, I. S. (2011). Principais causas de hospitalização de pacientes em hemodiálise no município de Guarapuava, Paraná, Brasil. Revista Gaúcha de Enfermagem. 
Research, Society and Development, v. 11, n. 2, e14711222269, 2022

(CC BY 4.0) | ISSN 2525-3409 | DOI: http://dx.doi.org/10.33448/rsd-v11i1.22269

Salimena, A. M. de O. et al. O cotidiano da mulher em hemodiálise. Revista Online de Pesquisa Cuidado é Fundamental, [s. l.], 2016. DOI 10.978/21755361.2016.v8i3.4636-4643. http://www.seer.unirio.br/index.php/cuidadofundamental/article/view/3664.

Santana, N. F.; Nobre, V. N. N.; Luz, L. K. T. da. Autocuidado com fístula arteriovenosa em terapia renal substitutiva. Revista Científica de Enfermagem, [s. l.], 2019.

Santana, M. B. A. et al. Autocuidado en personas con enfermedad renal crónica en hemodiálisis. Revista Gaúcha de Enfermagem, [s. l.], 2020. DOI 10.1590/1983-1447.2020.20190220. https://www.scielo.br/scielo.php?pid=S1983-14472020000100415\&script=sci_arttext\&tlng=es. Acesso em: 29 jan. 2021.

Sampaio, C. d. F., \& Guedes, M. V. C. (2012). Processo de enfermagem como estratégia no desenvolvimento de competência para o autocuidado. Acta Paulista Enfermagem.

Santos, I.i dos; Rocha, R. de P. F.; Berandinelli, L. M. M. Necessidades de orientação de enfermagem para o autocuidado de clientes em terapia de hemodiálise. Revista Brasileira de Enfermagem, [s. l.], 2011.

Santos, Bianca Pozza dos et al. Doença renal crônica: relação dos pacientes com a hemodiálise. Arquivo Brasileiro de Ciências da Saúde, [s. l.], 2017.

Santos, M. V. B. dos; Lira, G. G.; Fernandes, F. E.C. V. Adesão à medicação pelo paciente renal crônico em hemodiálise. Revista de Enfermagem UFPE Online, [s. l.], 2019. DOI 10.5205/1981-8963.2020.243294. https://pesquisa.bvsalud.org/portal/resource/pt/biblio-1096532.

Silva, Dejanilton Melo da et al. O corpo marcado pela fístula arteriovenosa: um olhar fenomenológico. Revista Brasileira de Enfermagem, [s. l.], 2018

Sociedade Brasileira de Nefrologia, S. (n.d.). http://www.sbn.org

Stefani, S. D., \& Barros, E. (2013). Clínica Médica: consulta rápida (4th ed.). Artmed.

Xavier, B. L. S., Santos, I. d., Almeida, R. F., Clos, A. C., \& Santos, M. T. d. (2014). Características individuais e clínicas de clientes com doença renal crônica em terapia renal substitutiva. Revista Enfermagem UERJ.

Xavier, B. L. S. et al. Promovendo autocuidado em clientes em hemodiálise: aplicação do diagrama de nola pender. Revista Online de Pesquisa Cuidado é Fundamental, [s. l.], 2017. DOI 10.9789/2175-5361.2017.v9i2.545-550. http://www.seer.unirio.br/index.php/cuidadofundamental/article/view/5968.

Xavier, C. A., Figueiredo, P. P. d., \& Canuso, L. D. d. S. (2020). Construção de tecnologia educativa digital para o autocuidado em insuficiência cardíaca. Ressearch, Society and Development.

Yang, Miao-miao et al. Self-Care Behavior of Hemodialysis Patients With Arteriovenous Fistula in China: A Multicenter, Cross-Sectional Study. Therapeutic Apheresis and Dialysis, [s. l.], p. 167-172, 2019. 